\title{
Comparison of Anatomical and Functional Outcomes of Intravitreal Dexamethasone Implant between Phakic and Pseudophakic Eyes with Diabetic Macular Edema
}

\author{
Havva Kaldırım¹, Fatma Savur', Ahmet Kırgız², Kürşat Atalay ${ }^{1}$ \\ ${ }^{1}$ Department of Ophthalmology, Bagcilar Training and Research Hospital, University of Health Sciences, Istanbul, Turkey \\ ${ }^{2}$ Department of Ophthalmology, Beyoglu Eye Training and Research Hospital, University of Health Sciences, Istanbul, Turkey
}

Purpose: To compare the effectiveness of dexamethasone (DEX) intravitreal implants for the treatment of diabetic macular edema between phakic and pseudophakic eyes after a follow-up.

Methods: A total of 79 eyes in 79 patients with diabetic macular edema who were insufficiently responsive to the previous anti-vascular endothelial growth factor treatment and applied intravitreal DEX implant were included in this retrospective study. The patients were divided into two groups according to their type of lenses: pseudophakic (group 1) and phakic (group 2). Best-corrected visual acuity, intraocular pressure (IOP), retinal nerve fiber layer thickness, and central macular thickness changes in the two groups were compared.

Results: Group 1 comprised 42 eyes in 42 patients with a mean age of $64.02 \pm 3.79$ years, while group 2 comprised 37 eyes in 37 patients with a mean age of $64.19 \pm 5.08$ years. In both groups, best-corrected visual acuity improved significantly with a significant decrease in central macular thickness $(p<0.001)$. In both lens types, the IOP values decreased significantly on the first day but subsequently increased significantly compared to baseline from one week to six months. Increases in IOP values were significantly higher in group 1 at the first week, 1st month, and 6th month compared with group 2. During follow-up, the inferior quadrant retinal nerve fiber layer showed a significant decrease in both groups.

Conclusions: In both phakic and pseudophakic eyes, intravitreal DEX implantation is an effective treatment method with low-risk complications. In our study, the pseudophakic group experienced a significantly earlier increase in IOP and at higher levels than did the phakic group. In light of these data, we suggest that in patients with pseudophakic eyes, follow-ups for IOP should be stricter and started earlier after intravitreal DEX treatment.

Key Words: Dexamethasone intravitreal implant, Diabetic macular edema, Phakic eye, Pseudophakic eye

Diabetic macular edema (DME) is a major cause of vision loss in patients with diabetes [1,2]. In the pathogenesis

Received: December 21, 2019 Final revision: May 19, 2020

Accepted: July 23, 2020

Corresponding Author: Havva Kaldırım, MD. Department of Ophthalmology, University of Health Sciences, Bagcilar Training and Research Hospital, Istanbul, Turkey. Tel: 90-212-440-4000, Fax: 90-212-440-4242, E-mail: istanbuleah30@saglik.gov.tr of DME, inflammatory cytokines, including intercellular adhesion molecule-1, interleukin-6, monocyte chemotactic protein-1, leukostasis, and vascular endothelial growth factor (VEGF) are responsible for disturbing the blood-retinal barrier and causing capillary leakage [3]. For a long time, therapeutic alternatives in DME involved laser photocoagulation. However, this procedure has a limited effect in restoring lost vision, which may be due to expanded retinal 
scars over time, decrease in vision, and contrast sensitivity [4]. Considering the pathogenesis of DME, alternative treatment methods have been developed to improve the quality of life of patients. Other commonly used treatment options for DME include intravitreal anti-VEGF and dexamethasone (DEX) implants $[5,6]$. Shorter action times and higher recurrence of macular edema limit the use of commonly used intraocular anti-VEGF injections [5]. Multiple factors in DME pathogenesis, suggest that effective treatment cannot be achieved only by inhibition of VEGF. Intravitreal corticosteroids are known to have anti-inflammatory, anti-apoptotic, antiedemat, and anti-angiogenic effects; intravitreal DEX implantation is an effective treatment option for DME [6]. Elevation in intraocular pressure (IOP) and the risk of cataract progression are the most common complications of intravitreal DEX implantation [7]. These complications limit the use of intravitreal DEX as the first-line treatment for DME. However, it is necessary to combine existing treatment options for resistant DME. There are many factors that affect the effectiveness of treatment options used in the treatment of a disease with complex pathogenesis, such as DME. Krohne et al. [8] investigated the effect of lens status on the pharmacokinetics of intravitreal VEGF inhibitor. They thought that diffusion of intravitreal drugs into the anterior chamber and then elimination of the eye through the Schlemm channel would be faster in pseudophakic eyes than in phakic eyes. Aqueous humor, intravitreal ranibizumab, or bevacizumab injection of phakic and pseudophakic patients were sampled at different time points, and drug or VEGF concentrations were measured. As a result, they thought that the lens condition had no effect on the duration of action of ocular pharmacokinetics and VEGF inhibitor drugs [8].

An intravitreal DEX implant (0.7 or $0.35 \mathrm{mg}$ ) (Ozurdex; Allergan, Irvine, CA, USA) consists of micronized DEX in a biodegradable copolymer of polylactic-co-glycolic acid, which slowly releases steroids into the vitreous over a period of about 6 months [6]. In 2014, based on the results of the MEAD study, the Food and Drug Administration and most European countries approved DEX implants for the treatment of DME [9].

Different studies have demonstrated that DEX implants may improve central macular thickness (CMT) and best-corrected visual activity (BCVA) in patients with DME $[10,11]$. There is not much evidence addressing the impact of lens type on the treatment of DME with DEX implant outcomes. Therefore, in the present study, we aimed to evaluate the anatomical and functional outcomes, including BCVA, CMT, IOP, and four-quadrant retinal nerve fiber layer (RNFL) thicknesses of patients treated with intravitreal DEX implant for DME and compare the results regarding the lens type of patients.

\section{Materials and Methods}

The study was performed in Health Science Univercity Bagcilar Training and Research Hospital, between January 2016 and January 2018. Ethical approval was obtained from the Local Clinical Research Ethics Committee T. C. Public Hospitals Institution, Bagcilar Training and Research Hospital Non Interventional Clinical Research Ethics Committee (project no. 2017/600). The study adhered to the tenets of the Declaration of Helsinki. Written informed consent for treatment was obtained from all patients before the treatment.

These data were retrospectively obtained from patient records. DME patients older than 18 years who did not respond to previous anti-VEGF treatments and who had 6-month follow-up data after intravitreal DEX implantation were included in the study. Patients included in the study, macular thickness was never CMT $<350 \mu \mathrm{m}$ during anti-VEGF injection. Patients with any history of previous complicated cataract surgery, naïve DME, neodymium-doped yttrium aluminum garnet (Nd-YAG) laser capsulotomy, vitreo-retinal surgery or laser treatment in the last 1 year, intravitreal DEX implant treatment, intravitreal triamcinolone acetonide, uveitis, glaucoma, or ocular hypertension were excluded from the study. Patients with incomplete follow-up data were also not included in the study. The patients were divided into two groups according to their type of lenses: pseudophakic (group 1) and phakic (group 2).

The age, sex, lens status of the patients, and previous treatments such as Nd-YAG laser capsulotomy, argon laser, or anti-VEGF were recorded. All recruited patients underwent a complete ophthalmic evaluation, including assessment of BCVA, IOP measurement by Goldman applanation tonometry, slit-lamp biomicroscopic evaluation, and indirect fundus ophthalmoscopy. The diagnosis of diabetic retinopathy was made by fundus examination and fundus fluorescein angiography. In addition, spectral domain optical 
Table 1. Pretreatment demographics features of patients

\begin{tabular}{lccc}
\hline & Group 1 ${ }^{*}(\mathrm{n}=42)$ & Group 2 $^{\dagger}(\mathrm{n}=37)$ & $p_{\text {-value }}^{\ddagger}$ \\
\hline Sex (male : female) & $19: 23: 19$ & 18 & 0.46 \\
Age (yr) & $64.02 \pm 3.79$ & $64.19 \pm 5.08$ & 0.87 \\
The mean duration of diabetes (yr) & $22.5 \pm 3.1$ & $22.86 \pm 2.54$ & 0.89 \\
Hemoglobin A1c levels (mmol/mol) & $7.91 \pm 0.16$ & $7.86 \pm 0.13$ & 0.78 \\
Previous anti-VEGF injection number & $5.2 \pm 1.4$ & $6.3 \pm 2.1$ & 0.001 \\
Eyes treated with argon laser more than one year ago & $10(23.8)$ & $7(18.9)$ & 0.58 \\
\hline
\end{tabular}

Values are presented as means \pm standard deviation or number (\%).

$\mathrm{VEGF}=$ vascular endothelial growth factor.

"Pseudophakic; ${ }^{*}$ Phakic; ${ }^{*}$ The significance level of comparison between 2 groups (independent samples $t$-test).

coherence tomography (Retinascan RS-3000; NIDEK, Gamagori, Japan) was performed in all cases. CMT and the superior, inferior, nasal, and temporal quadrant peripapillary RNFL thicknesses were determined using spectral domain optical coherence tomography. BCVA was measured in Snellen decimals and converted to the logarithm of the minimal angle of resolution for statistical analyses.

A sustained-release DEX $0.7 \mathrm{mg}$ intravitreal implant (IDI; Ozurdex, Allergan) was injected intravitreally with its special applicator, from the pars plana region, in the operating room under sterile conditions. After the injection, topical antibiotics were administered five times a day for five days. IOP was obtained on day 1, first week, months 1 , 2, 3, and 6, respectively. Four quadrant RNFL thicknesses were obtained at 1,2,3, and 6 months while BCVA and CMT were measured at 1,3 , and 6 months, respectively. Topical anti-glaucomatous agents were started in patients who had IOP $\geq 22 \mathrm{mmHg}$ at any control visit. The patients were followed up for 6 months. Hemoglobin Alc values before treatment were also recorded.

The statistical analyses were performed using the software IBM SPSS Statistics ver. 22.00 (IBM Corp., Armonk, NY, USA). Descriptive statistics were performed. Numerical variables are expressed as mean \pm standard deviation, and categorical variables are expressed as frequencies. The distribution of variables was investigated using the Kolmogorov-Smirnov test. The results at different time intervals of the same group were compared using repeated measures ANOVA (with a post-hoc Tukey test), while the results of the two groups were compared using the chi-square test and independent samples $t$-test. Statistical significance was set at $p<0.05$.

\section{Results}

A total of 79 eyes in 79 diabetic patients were enrolled in the study. Group 1 (pseudophakic), comprised of 42 eyes in 42 patients (19 male and 23 female) with a mean age of (mean \pm standard deviation) $64.02 \pm 3.79$ years, while group 2 (phakic) comprised of 37 eyes in 37 patients (18 male and 19 female) with a mean age of $64.19 \pm 5.08$ years. There was no statistically significant difference between the two groups regarding age ( $p=0.87)$ or sex $(p=0.46)$. In groups 1 and 2 , the mean duration of diabetes was 22.95 \pm 3.01 and 22. $86 \pm 2.54$ years $(p=0.89)$, respectively, and pretreatment hemoglobin Alc levels $(\mathrm{mmol} / \mathrm{mol})$ were 7.91 \pm 0.16 and $7.86 \pm 0.13(p=0.78)$, respectively. Number of patients who received previous anti-VEGF injections were $5.2 \pm 1.4$ in group 1 and $6.3 \pm 2.1$ in group $2(p=0.001)$. Moreover, 10 (23.8\%) eyes in group 1 and 7 (18.9\%) eyes in group 2 were treated using an argon laser more than 1 year ago $(p=0.58)$ (Table 1). Nd-YAG laser capsulotomy was not performed in any case.

In group $1, C M T$ was $447 \pm 42 \mu \mathrm{m}$ prior to DEX implant, $299 \pm 21 \mu \mathrm{m}$ at the $1 \mathrm{st}, 339 \pm 28 \mu \mathrm{m}$ at the $3 \mathrm{rd}$, and $322 \pm$ $22 \mu \mathrm{m}$ at the 6th month, respectively. CMT in group 2 at the baseline visit was $437 \pm 26 \mu \mathrm{m}, 297 \pm 25 \mu \mathrm{m}$ at the 1st, $314 \pm 28 \mu \mathrm{m}$ at the $3 \mathrm{rd}$, and $312.89 \pm 39 \mu \mathrm{m}$ at the 6 th month, respectively. In groups 1 and 2, the changes in CMT were statistically significant at the 1st, 3rd and 6th months when compared to baseline visit values $(p 1<0.001)$. However, at the end of the 3rd month, the CMT value was significantly different between the two groups $(p 2=0.01)$. In all other time periods, there were no significant differences between the two groups regarding CMT values (Table 2). 
Table 2. Alterations in CMT and BCVA during follow-ups in both groups

\begin{tabular}{lccc}
\hline & Group 1 $(\mathrm{n}=42)$ & Group 2 (n=37) & $p 2$-value \\
\hline Baseline CMT $(\mu \mathrm{m})$ & $447.02 \pm 4.80$ & $437.08 \pm 25.52$ & 0.20 \\
CMT-1st month & $299.38 \pm 2.93$ & $296.73 \pm 19.99$ & 0.57 \\
CMT-3rd month & $338.71 \pm 28.26$ & $314.30 \pm 27.98$ & 0.01 \\
CMT-6th month & $322.33 \pm 21.85$ & $312.89 \pm 39.33$ & 0.37 \\
$p$ 1-value & $<0.001$ & $<0.001$ & 0.49 \\
Baseline BCVA (logMAR) & $0.56 \pm 0.10$ & $0.58 \pm 0.08$ & 0.65 \\
BCVA-1st month & $0.29 \pm 0.09$ & $0.29 \pm 0.08$ & 0.04 \\
BCVA-3rd month & $0.32 \pm 0.09$ & $0.30 \pm 0.08$ & 0.34 \\
BCVA-6th month $^{\dagger}$ & $0.32 \pm 0.08$ & $0.34 \pm 0.07$ & $<0.001$ \\
$p$-value
\end{tabular}

Values are presented as means \pm standard deviation.

$\mathrm{CMT}=$ central macular thickness; BCVA = best-corrected visual acuity.

${ }^{*}$ The significance level of analysis performed between groups (independent samples $t$-test); ${ }^{\dagger}$ The significance level of intra-group analysis (repeated measures ANOVA with a post-hoc Tukey test).

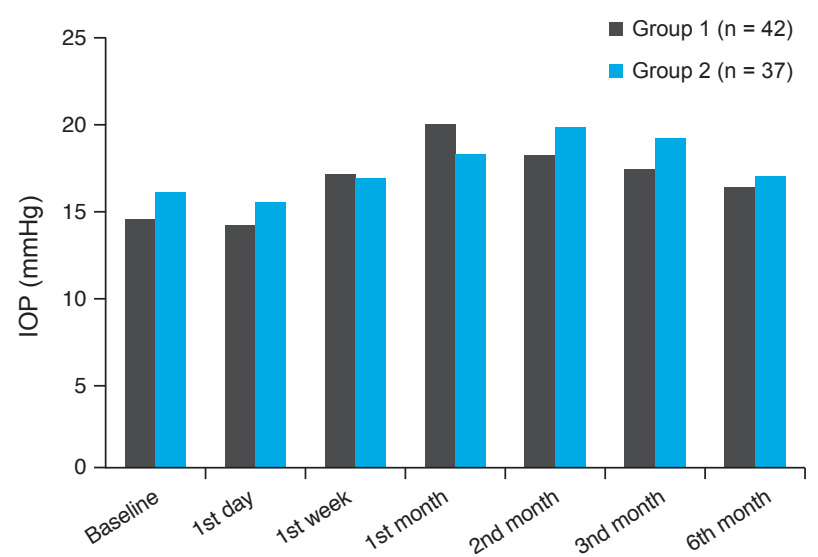

Fig. 1. Alterations in intraocular pressure (IOP) values among two groups during study period. The maximum mean IOP values were obtained on the 1st month in group 1 and on the 2 nd month of treatment in group 2.

In group 1, BCVA (logarithm of the minimal angle of resolution) was $0.56 \pm 0.10$ prior to DEX implant, $0.29 \pm 0.09$ at the 1st, $0.32 \pm 0.09$ at the 3rd and $0.32 \pm 0.08$ at the 6th month. In group 2, BCVA was $0.58 \pm 0.08$ at baseline, 0.29 \pm 0.08 at the 1 st month, $0.30 \pm 0.08$ at the 3rd month, and $0.34 \pm 0.07$ at the 6 th month, respectively. In groups 1 and 2 , the changes in BCVA were statistically significant at the 1st, 3rd, and 6th months compared to baseline visit values ( $p 1<$ 0.001). At the end of the $3 \mathrm{rd}$ month, the BCVA value was significantly different between the two groups $(p 2=0.04)$. In all other times, there were no significant differences be-

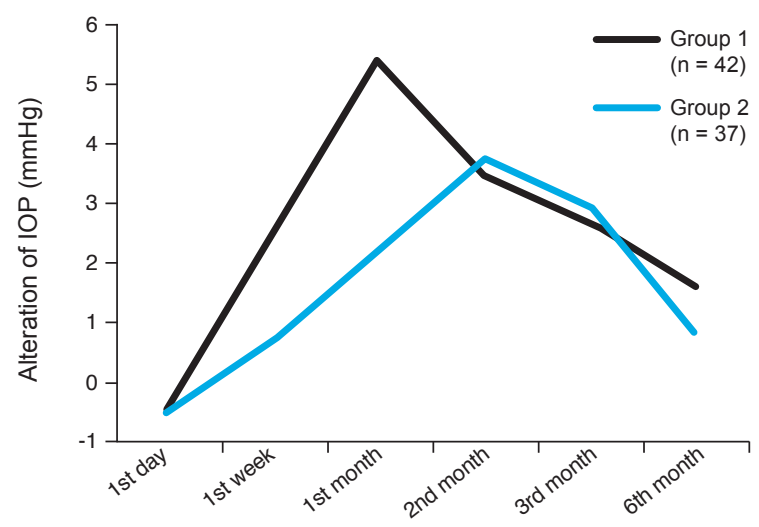

Fig. 2. Alterations in intraocular pressure (IOP) compared with the pretreatment values in both groups during follow-up period. IOP values in both groups decreased significantly on the first day, but IOP values were significantly higher than the baseline values from the first week to the 6th month.

tween two groups regarding BCVA values (Table 2).

Alterations in IOP values between the two groups during the study period are summarized in Fig. 1, and alterations in IOP from the baseline are summarized in Table 3 and Fig. 2. In both lens types, the IOP values decreased significantly on the first day, but from the first week to the 6th month, IOP values were significantly higher than the baseline values. The maximum mean IOP values were obtained on the 1st month in group 1 and on the 2nd month of treatment in group 2. At the end of the first week in five (11.9\%) eyes of group 1 and in two (5.4\%) eyes of group 2, IOP val- 
Table 3. Differences in IOP values at different time intervals compared with the pretreatment IOP

\begin{tabular}{lccc}
\hline & $\begin{array}{c}\text { Group 1 } \\
(\mathrm{n}=42)\end{array}$ & $\begin{array}{c}\text { Group 2 } \\
(\mathrm{n}=37)\end{array}$ & $p$-value $^{*}$ \\
\hline 1st day $(\mathrm{mmHg})$ & $-0.42 \pm 0.54$ & $-0.45 \pm 0.50$ & 0.80 \\
1st week $(\mathrm{mmHg})$ & $2.61 \pm 1.92$ & $0.72 \pm 0.99$ & 0.001 \\
1st month (mmHg) & $5.45 \pm 4.49$ & $2.18 \pm 2.69$ & 0.001 \\
2nd month (mmHg) & $3.47 \pm 2.78$ & $3.78 \pm 2.71$ & 0.62 \\
3rd month (mmHg) & $2.69 \pm 2.60$ & $2.97 \pm 2.52$ & 0.71 \\
6th month (mmHg) & $1.66 \pm 1.94$ & $0.83 \pm 1.32$ & 0.03 \\
\hline
\end{tabular}

Values are presented as means \pm standard deviation. $\mathrm{IOP}=$ intraocular pressure

"The significance level of comparison between two groups (independent samples $t$-test).

ues were higher than $22 \mathrm{mmHg}$. IOP values higher than 22 $\mathrm{mmHg}$ in eyes of group 1 an d 2 were seen in five (11.9\%) and one (2.7\%), respectively in the first month; in two (4.8\%) and four (10.8\%), respectively in 2nd month; in one (2.4\%) and $(10.8 \%)$ eyes, respectively in $3 \mathrm{rd}$ month and in no patients in both groups at the end of 6th month. Anti-glaucomatous treatment was required in $13(30.9 \%)$ patients in group 1 and in $10(27.0 \%)$ patients in group 2 during the treatment period; however, at the end of study, one $(2.7 \%)$ patient in group 1 and one patient (3.4\%) in group 2 ( $p=$ $0.26)$ continued anti-glaucomatous treatment. None of the patients showed a resistant increase in IOP requiring surgery. Increase in IOP values were significantly higher in group 1 at the end of the 1st week and 6th month compared with group 2 ( $p=0.001, p=0.03$, respectively). The number of patients who had $\mathrm{a} \geq 10 \mathrm{mmHg}$ increase in IOP from baseline during the follow-up period was: eight (19.0\%) patients in group 1 and seven (18.9\%) patients in group $2(p$ $=0.86$ ).

Alterations in four quadrant RNLF values during the treatment period were also recorded and were compared with the baseline values in each group (Table 4). There was no statistically significant difference in four-quadrant basal RNFL between the two groups ( $p=0.43, p=0.44, p=0.78$, $p=0.49$, respectively). As such, in group 1 patients who had a $\geq 10$ - $\mathrm{mmHg}$ increase in IOP from baseline, inferior RNLF was significantly lower at the 2nd, 3rd, and 6th month measurements than at baseline $(p=0.01)$. Similarly, in group 2 patients who had a $\geq 10-\mathrm{mmHg}$ increase in IOP from baseline, inferior RNLF was significantly lower at
Table 4. Alterations in four quadrants RNFL during follow-ups in both groups

\begin{tabular}{|c|c|c|c|}
\hline & $\begin{array}{l}\text { Group } 1 \\
(\mathrm{n}=42)\end{array}$ & $\begin{array}{l}\text { Group } 2 \\
(\mathrm{n}=37)\end{array}$ & $p 2$-value \\
\hline $\begin{array}{l}\text { Baseline inferior } \\
\text { RNFL }(\mu \mathrm{m})\end{array}$ & $98.05 \pm 8.65$ & $96.97 \pm 8.90$ & 0.43 \\
\hline 1st month & $96.14 \pm 7.92$ & $96.95 \pm 8.89$ & 0.88 \\
\hline 2nd month & $95.57 \pm 7.75$ & $96.24 \pm 8.61$ & 0.58 \\
\hline 3rd month & $95.48 \pm 7.75$ & $95.70 \pm 8.18$ & 0.82 \\
\hline 6th month & $95.38 \pm 7.89$ & $95.03 \pm 7.98$ & 0.78 \\
\hline$p 1$-value ${ }^{\dagger}$ & 0.01 & 0.01 & \\
\hline $\begin{array}{l}\text { Baseline superior } \\
\text { RNFL }(\mu \mathrm{m})\end{array}$ & $92.88 \pm 7.33$ & $94.92 \pm 8.57$ & 0.44 \\
\hline 1 st month & $92.07 \pm 6.90$ & $94.92 \pm 8.57$ & 0.52 \\
\hline 2nd month & $91.88 \pm 6.75$ & $94.59 \pm 8.37$ & 0.22 \\
\hline 3rd month & $91.93 \pm 6.78$ & $94.27 \pm 8.08$ & 0.31 \\
\hline 6th month & $91.88 \pm 6.92$ & $93.84 \pm 7.80$ & 0.46 \\
\hline$p 1-$ value $^{\dagger}$ & 0.22 & 0.14 & \\
\hline $\begin{array}{l}\text { Baseline nasal RNFL } \\
\quad(\mu \mathrm{m})\end{array}$ & $71.81 \pm 3.79$ & $71.03 \pm 2.77$ & 0.78 \\
\hline 1 st month & $71.69 \pm 3.65$ & $71.08 \pm 2.80$ & 0.82 \\
\hline 2nd month & $71.69 \pm 3.65$ & $71.08 \pm 2.80$ & 0.86 \\
\hline 3rd month & $71.62 \pm 3.62$ & $71.03 \pm 2.80$ & 0.82 \\
\hline 6th month & $71.64 \pm 3.59$ & $70.97 \pm 2.76$ & 0.88 \\
\hline$p 1$-value ${ }^{\dagger}$ & 0.79 & 0.81 & \\
\hline $\begin{array}{l}\text { Baseline temporal } \\
\text { RNFL }(\mu \mathrm{m})\end{array}$ & $67.64 \pm 3.67$ & $69.24 \pm 2.10$ & 0.49 \\
\hline 1st month & $67.60 \pm 3.63$ & $69.24 \pm 2.10$ & 0.56 \\
\hline 2nd month & $67.60 \pm 3.63$ & $69.24 \pm 2.10$ & 0.56 \\
\hline 3rd month & $67.55 \pm 3.58$ & $69.30 \pm 2.15$ & 0.47 \\
\hline 6th month & $67.55 \pm 3.60$ & $69.30 \pm 2.17$ & 0.47 \\
\hline$p 1$-value ${ }^{\dagger}$ & 0.82 & 0.91 & \\
\hline
\end{tabular}

Values are presented as means \pm standard deviation.

RNFL $=$ retinal nerve fiber length.

"The significance level of analysis performed between groups (independent samples $t$-test); ${ }^{+}$The significance level of intra-group analysis (repeated measures ANOVA with a post-hoc Tukey test).

the 6th month than at baseline $(p=0.01)$. There were no significant alterations in either group regarding the superior, nasal, or temporal RNLF values during 6 months follow-up after intravitreal DEX implantation.

Additional treatment applied between 4th and 6th months of follow-up was also recorded. Overall, 33 eyes (78.6\%) in group 1 and 22 eyes (59.5\%) in group 2 received an additional anti-VEGF treatment $(p=0.34)$. 


\section{Discussion}

In this study, we compared the anatomical and functional outcomes of pseudophakic and phakic eyes with DME after single intravitreal DEX treatment and determined that there were no significant effects of lens type on outcomes at the end of the 6th month. Moreover, in the intra-group analysis, we also determined that in both lens types, CMT values decreased and BCVA values improved significantly in both groups at all-time points, compared with the pre-injection data, supporting that intravitreal DEX implant was effective in this group of patients, who were partially responsive to previous anti-VEGF treatments. When four quadrant RNLF values during the treatment period were evaluated, only inferior RNLF values showed a significant alteration from the baseline values. Notably, from the first week until the 6th month of treatment, IOP values were significantly higher than the baseline values. However, at the end of the 6th month, one (2.7\%) patient in group 1 and one patient (3.4\%) in group 2 continued the anti-glaucomatous treatment. An important finding of this study is that, in the pseudophakic eye group, increases in IOP values were earlier and more prominent compared to the phakic eyes.

To the best of our knowledge, data comparing the lens types regarding intravitreal DEX implant with DME is limited. Previous studies have suggested that intravitreal corticosteroid treatment may be more effective in pseudophakic eyes than in phakic eyes $[12,13]$. In the current literature, there are some studies regarding the efficacy of intravitreal DEX implants in DME. In a retrospective study of 186 eyes in 165 patients, Moon et al. [14], reported that the mean BCVA improved until 3 months and then decreased up to 6 months of follow-up, and they reported that this treatment was more effective in pseudophakic eyes. In the same study, they also reported that $48.9 \%$ of the eyes received additional treatment with anti-VEGF or DEX implants. Unsal et al. [15], investigated the efficacy of intravitreal DEX implants in 46 eyes with DME that were unresponsive to previous treatments and reported that BCVA improved significantly in the first 4 months, and CMT decreased significantly in the first 3 months. In a recent meta-analysis of 3,859 patients from 15 studies, intravitreal DEX implant was found to be associated with significant mean improvement in BCVA in patients with DME who have a suboptimal response to anti-VEGF ther- apy [16]. Totan et al. [17], also reported that the therapeutic efficacy of intravitreal DEX implant decreases between 3rd and 6th months after injection. In our study, the best BCVA values were obtained in the 1st month in both groups. Similarly, the lowest CMT values were also determined at the end of the 1st month. In both groups at the end of the 3rd month, BCVA and CMT values were significantly improved compared with the pretreatment values. However, the early improvements in BCVA and CMT started to decrease in the 3rd month in the pseudophakic group. Although data on the effects of lens type on BCVA and CMT are limited, its mechanism is not well known. We suggest that this difference may be associated with alterations in the structure and dynamics of the vitreous after cataract surgery, affecting DEX implant secretion. In this study, between the 3rd and 6th months of follow-up, additional anti-VEGF treatments were required in $78.6 \%$ of group 1 and $59.5 \%$ of group 2 patients.

The most common complications associated with intravitreal DEX implantation are elevations in IOP and cataract progression. The pathophysiological mechanism of IOP elevation is the modulation of steroids on the trabecular meshwork (TM) by increasing deposits at TM and suppressing the phagocytic effect in TM [18-20]. Recently, several studies have evaluated the degree and management of post injection IOP elevations induced by DEX [21,22]. In a randomized, multicenter study with 3 years of follow-up on 1,048 patients diagnosed with DME, $\geq 10-\mathrm{mmHg}$ increases in IOP from baseline was determined in $27.7 \%$ of patients treated with $0.7 \mathrm{mg}$ DEX implant [22]. However, they reported that IOP-lowering medication was required in $41.5 \%$ of patients, while only one patient $(0.3 \%)$ required glaucoma surgery [22]. In our study, about $19 \%$ of patients in both groups showed $\geq 10$-mmHg increase in IOP levels from baseline. Anti-glaucomatous treatment was required in $13(30.9 \%)$ patients in group 1 and in $10(27.0 \%)$ patients in group 2 during the treatment period; however, at the end of the study, one (2.7\%) patient in group 1 and one patient (3.4\%) in group 2 continued treatment. In group 1, the increase in IOP values was mainly observed in 1st month, with four eyes having an IOP of higher than $30 \mathrm{mmHg}$, while, in group 2, those elevations were reported mainly in the 2nd and 3rd months, and an IOP of $>30 \mathrm{mmHg}$ was not observed in any patient. Surgery was not required in any patient. In both lens types, during the 6th month follow-up, the mean IOP values were still higher than the baseline 
values. Interestingly, we also reported significantly higher increase of IOP in the pseudophakic group than in the phakic group at the end of the first week and 1st month. We explain this effect by suggesting that in pseudophakic eyes, DEX particles pass through the anterior chamber more rapidly and may alter the TM structure earlier. Considering these differences in IOP elevations, in patients with pseudophakic eyes, follow-ups for IOP increases should be started earlier and should be strictly monitored after intravitreal DEX implantation.

Despite the promising therapeutic benefit of DEX implants in the treatment of macular edema, studies have described temporary IOP spikes as a common adverse event in up to $27 \%$ to $32 \%$ of the patients [9,23]. Increased IOP, experimentally induced in animal models, has demonstrated similar ultrastructure retinal ganglion cell changes as seen in optic nerve fibers in postmortem human eyes that have glaucoma [24]. Although IOP elevation is considered a risk factor for optic nerve damage and is the only modifiable risk factor for glaucoma, the exact pathogenesis of glaucoma is not fully understood and is likely to be multifactorial [25]. According to a recent systematic review by Bucolo et al. [26], the vast majority of steroid responders who receive DEX implant can be managed with topical IOP reducing drops and rarely require incisional glaucoma surgeries. Nevertheless, DEX implants must be used with caution, and IOP checks must be performed diligently, especially in patients with ocular history of glaucoma. Whether optic nerve damage is a result of DEX-related IOP spikes or the natural progression of the vitreoretinal disease, remains unclear. In addition, type 2 diabetes mellitus may cause RNFL loss with systemic complications, with or without retinopathy. Garcia-Martin et al. [27], showed RNFL loss in patients with type 2 diabetes mellitus without retinopathy. This shows that multiple factors cause loss of RNFL in patients with diabetes mellitus. Determination of the RNFL is important since it is defined as an early sign of glaucoma. Some studies have shown that RNFL thinning starts primarily in the inferior quadrant [28-30]. To the best of our knowledge, this is the first study to evaluate the effects of intravitreal DEX implantation on RNLF in patients with DME. However, there are a limited number of studies evaluating the effects of this treatment modality on RNFL in patients with branch retinal vein occlusion (BRVO). Ayar et al. [31], evaluated the effects of intravitreal DEX implant on RNFL thickness in patients with BRVO in a 6-month period and reported that there were no significant changes in mean RNFL values postoperatively compared to baseline values in all quadrants. On the other hand, Bulut et al. [32], reported that the mean IOP was significantly increased from baseline at 1st day, 1st week, and 1st month visits, and inferior quadrant peripapillary RNFL thickness was significantly reduced at the 6th month compared to at baseline in patients with BRVO-related macular edema. Similarly, we also determined a significant decrease in inferior peripapillary RNFL thickness in both groups at the end of the 6th month. In this study, patients with decrease in RNLF thickness were those with an increase in IOP value greater than $10 \mathrm{mmHg}$. In the pseudophakic group, the decrease in RNLF thickness was determined in the 1st month, while this decrease was determined in the 3rd month in the phakic group. This earlier alteration may be associated with earlier IOP increase in group 1 compared with group 2. The greatest decrease in RNLF thickness was determined in four patients with an IOP increase of more than 30 $\mathrm{mmHg}$ in group 1. In fact, considering this data in the first 3 months, to prevent a possible decrease in RNLF thickness, prophylactic topical anti-glaucomatous agents may be advised, but larger prospective studies are warranted to determine the timing of drug prescription in patients with DME.

DEX implant treatment is less commonly associated with migration into the anterior chamber, requiring surgical correction, endophthalmitis, and retinal or vitreous hemorrhage due to retinal impact during injection [33-35]. None of the complications was reported in this study. Posterior subcapsular cataracts were observed in only two patients in group 2.

This study has some limitations that need to be mentioned. The first is the retrospective design of the study, and secondly, the cosmopolite nature of patients in terms of the previous and additional treatments could have altered our results.

In conclusion, in light of these data, we can suggest that intravitreal DEX implantation is an effective treatment method with low risk of complications in both phakic and pseudophakic eyes; moreover, lens type does not affect the outcomes of intravitreal DEX implantation at the end of the 6th month in patients with DME. However, increases in IOP values were observed in the pseudophakic group at significantly higher levels and occurred earlier compared 
with the phakic eyes without any significant differences in the four quadrant RNFL thicknesses except in inferior RNFL. Based on these data, we suggest that in patients with pseudophakic eyes, follow-ups for increase in IOP after intravitreal DEX implantation should be started earlier and be more strict. Larger prospective studies with longer follow-up periods are warranted to elucidate the functional and anatomical outcomes after intravitreal DEX implantation in patients with DME.

\section{Conflict of Interest}

No potential conflict of interest relevant to this article was reported.

\section{References}

1. Williams R, Airey M, Baxter H, et al. Epidemiology of diabetic retinopathy and macular oedema: a systematic review. Eye (Lond) 2004;18:963-83.

2. Yau JW, Rogers SL, Kawasaki R, et al. Global prevalence and major risk factors of diabetic retinopathy. Diabetes Care 2012;35:556-64.

3. Lattanzio R, Cicinelli MV, Bandello F. Intravitreal steroids in diabetic macular edema. Dev Ophthalmol 2017;60:78-90.

4. Qian TW, Zhao MY, Li XX, Xu X. Efficiency and safety of laser photocoagulation with or without intravitreal ranibizumab for treatment of diabetic macular edema: a systematic review and Meta-analysis. Int J Ophthalmol 2017;10:113443.

5. Wu Y, Ai P, Ai Z, Xu G. Subthreshold diode micropulse laser versus conventional laser photocoagulation monotherapy or combined with anti-VEGF therapy for diabetic macular edema: a Bayesian network meta-analysis. Biomed Pharmacother 2018;97:293-9.

6. Chang-Lin JE, Attar M, Acheampong AA, et al. Pharmacokinetics and pharmacodynamics of a sustained-release dexamethasone intravitreal implant. Invest Ophthalmol Vis Sci 2011;52:80-6.

7. Meyer LM, Schonfeld CL. Secondary glaucoma after intravitreal dexamethasone $0.7 \mathrm{mg}$ implant in patients with retinal vein occlusion: a one-year follow-up. J Ocul Pharmacol Ther 2013;29:560-5.

8. Krohne TU, Muether PS, Stratmann NK, et al. Influence of ocular volume and lens status on pharmacokinetics and duration of action of intravitreal vascular endothelial growth factor inhibitors. Retina 2015;35:69-74.

9. Boyer DS, Yoon YH, Belfort R Jr, et al. Three-year, randomized, sham-controlled trial of dexamethasone intravitreal implant in patients with diabetic macular edema. Ophthalmology 2014;121:1904-14.

10. Guigou S, Pommier S, Meyer F, et al. Efficacy and safety of intravitreal dexamethasone implant in patients with diabetic macular edema. Ophthalmologica 2015;233:169-75.

11. Pacella E, Vestri AR, Muscella R, et al. Preliminary results of an intravitreal dexamethasone implant (Ozurdex) in patients with persistent diabetic macular edema. Clin Ophthalmol 2013;7:1423-8.

12. Diabetic Retinopathy Clinical Research Network, Elman MJ, Qin H, et al. Intravitreal ranibizumab for diabetic macular edema with prompt versus deferred laser treatment: three-year randomized trial results. Ophthalmology 2012;119:2312-8.

13. Blumenkranz MS, Haller JA, Kuppermann BD, et al. Correlation of visual acuity and macular thickness measured by optical coherence tomography in patients with persistent macular edema. Retina 2010;30:1090-4.

14. Moon BG, Lee JY, Yu HG, et al. Efficacy and safety of a dexamethasone implant in patients with diabetic macular edema at tertiary centers in Korea. J Ophthalmol 2016;2016: 9810270.

15. Unsal E, Eltutar K, Sultan P, et al. Efficacy and safety of intravitreal dexamethasone implants for treatment of refractory diabetic macular edema. Korean J Ophthalmol 2017;31:115-22.

16. Khan Z, Kuriakose RK, Khan M, et al. Efficacy of the intravitreal sustained-release dexamethasone implant for diabetic macular edema refractory to anti-vascular endothelial growth factor therapy: meta-analysis and clinical implications. Ophthalmic Surg Lasers Imaging Retina 2017;48:1606.

17. Totan Y, Guler E, Guragac FB. Dexamethasone intravitreal implant for chronic diabetic macular edema resistant to intravitreal bevacizumab treatment. Curr Eye Res 2016;41:10713.

18. Clark AF, Brotchie D, Read AT, et al. Dexamethasone alters F-actin architecture and promotes cross-linked actin network formation in human trabecular meshwork tissue. Cell Motil Cytoskeleton 2005;60:83-95.

19. Zhou L, Li Y, Yue BY. Glucocorticoid effects on extracel- 
lular matrix proteins and integrins in bovine trabecular meshwork cells in relation to glaucoma. Int $\mathrm{J} \mathrm{Mol} \mathrm{Med}$ 1998;1:339-46.

20. Johnson DH, Bradley JM, Acott TS. The effect of dexamethasone on glycosaminoglycans of human trabecular meshwork in perfusion organ culture. Invest Ophthalmol Vis Sci 1990;31:2568-71.

21. Chin EK, Almeida DR, Velez G, et al. Ccular hypertension after intravitreal dexamethasone (Ozurdex) sustained-release implant. Retina 2017;37:1345-51.

22. Maturi RK, Pollack A, Uy HS, et al. Intraocular pressure in patients with diabetic macular edema treated with dexamethasone intravitreal implant in the 3-year mead study. Retina 2016;36:1143-52.

23. Capone A Jr, Singer MA, Dodwell DG, et al. Efficacy and safety of two or more dexamethasone intravitreal implant injections for treatment of macular edema related to retinal vein occlusion (Shasta study). Retina 2014;34:342-51.

24. Quigley HA, McKinnon SJ, Zack DJ, et al. Retrograde axonal transport of BDNF in retinal ganglion cells is blocked by acute IOP elevation in rats. Invest Ophthalmol Vis Sci 2000;41:3460-6.

25. Boland MV, Ervin AM, Friedman DS, et al. Comparative effectiveness of treatments for open-angle glaucoma: a systematic review for the U.S. Preventive Services Task Force. Ann Intern Med 2013;158:271-9.

26. Bucolo C, Gozzo L, Longo L, et al. Long-term efficacy and safety profile of multiple injections of intravitreal dexamethasone implant to manage diabetic macular edema: A systematic review of real-world studies. J Pharmacol Sci 2018;138:219-32.
27. Garcia-Martin E, Cipres M, Melchor I, et al. Neurodegeneration in patients with type 2 diabetes mellitus without diabetic retinopathy. J Ophthalmol 2019;2019:1825819.

28. Pieroth L, Schuman JS, Hertzmark E, et al. Evaluation of focal defects of the nerve fiber layer using optical coherence tomography. Ophthalmology 1999;106:570-9.

29. Schuman JS, Pedut-Kloizman T, Hertzmark E, et al. Reproducibility of nerve fiber layer thickness measurements using optical coherence tomography. Ophthalmology 1996;103:1889-98.

30. Quigley HA, Addicks EM. Quantitative studies of retinal nerve fiber layer defects. Arch Ophthalmol 1982;100:807-14.

31. Ayar O, Alpay A, Koban Y, et al. The effect of dexamethasone intravitreal implant on retinal nerve fiber layer in patients diagnosed with branch retinal vein occlusion. Curr Eye Res 2017;42:1287-92.

32. Bulut MN, Ozerturk Y, Calli U, et al. Evaluation of peripapillary nerve fiber layer after dexamethasone implantation (Ozurdex) in branch retinal vein occlusions. $J$ Ophthalmol 2016;2016:2050796.

33. Khurana RN, Appa SN, McCannel CA, et al. Dexamethasone implant anterior chamber migration: risk factors, complications, and management strategies. Ophthalmology 2014;121:67-71.

34. Esen E, Sizmaz S, Demircan N. Two cases of acute endophthalmitis after intravitreal dexamethasone implant injection. Retin Cases Brief Rep 2016;10:154-6.

35. Kucukevcilioglu M, Eren M, Yolcu U, Sobaci G. Acute retinal necrosis following intravitreal dexamethasone (Ozurdex) implant. Arq Bras Oftalmol 2015;78:118-9. 